\title{
On the Impact of Shadowing on the Performance of Cooperative Medium Access Control Protocols
}

\author{
M. Di Renzo*, J. Alonso-Zarate ${ }^{\dagger}$, L. Alonso ${ }^{\ddagger}$, Ch. Verikoukis ${ }^{\dagger}$ \\ *Laboratory of Signals and Systems (L2S), UMR 8506 CNRS - SUPELEC - Univ Paris-Sud, 3 rue Joliot-Curie, 91192 Gif-sur-Yvette, France \\ $\dagger$ Centre Tecnològic de Telecomunicacions de Catalunya (CTTC), 08860 Castelldefels, Barcelona, Spain \\ $\ddagger$ Dept. of Signal Theory and Communications, Universitat Politècnica de Catalunya (UPC), 08860 Castelldefels, Barcelona, Spain \\ E-Mail: marco.direnzo@1ss.supelec.fr, \{jesus.alonso, cveri\}@cttc.es, luisg@tsc.upc.edu
}

\begin{abstract}
Accurate representation of the physical layer is instrumental for a sound design and optimization of Medium Access Control (MAC) protocols for cooperative wireless networks. However, the vast majority of MAC protocols are designed and analyzed by considering simplified physical layer and channel models, which often lead to too optimistic performance predictions. In particular, even though many experimental activities have showcased the important role played by shadow-fading, most protocols are designed and evaluated by taking into account only the transmission distance (circular coverage model) or only the fast-fading. Motivated by the proved unsuitability of these models, the contribution of this paper is threefold: $i)$ we provide important considerations on how to adequately include the effect of shadowing into the design of MAC protocols for cooperative networks; ii) we provide an analytical framework to determine the subset of active relays in order to meet a given Quality-ofService (QoS) requirement; and iii) we study, through analysis and simulation, the performance of a promising MAC protocol for cooperative networks, which is called Persistent Relay Carrier Sensing Multiple Access (PRCSMA), by explicitly taking into account the effect of shadowing. Our study shows that shadowing can dramatically change system and protocol performance.
\end{abstract}

\section{INTRODUCTION}

Automatic Repeat reQuest (ARQ) mechanisms are extensively exploited by many link-layer protocols to reduce the packet error probability in communication networks. However, when applied to wireless networks, the effectiveness of ARQ schemes highly depends on the coherence time of the wireless channel: the longer the coherence time is, the less effective the ARQ mechanism is [1]. In fact, a longer coherence time implies that subsequent retransmitted packets might experience almost the same channel conditions, thus resulting in consecutive failures. To overcome this limitation, in [2] the authors have recently proposed an improved ARQ mechanism, which exploits distributed cooperation among nearby network nodes to improve the performance of link-layer protocols. The proposal in [2] is today known as Cooperative ARQ (C-ARQ) scheme, and it foresees that whenever a packet is received with insufficient quality, a retransmission can be requested not only to the original source node, but also to network nodes that are nearby the destination, which might have overheard the original transmission (due to the broadcast nature of the wireless channel) and might be willing to relay independent received versions of the same packet and provide an inherent form of spatial diversity, which is not affected by temporal correlation of fading. Recent results have shown the benefits of C-ARQ as opposed to non-cooperative schemes [3].

Research leading to these results has received funding from the research projects, CO2GREEN (TEC2010-20823), CENTENO (TEC2008-06817-C02-02), and GREENET (PITN-GA-2010-264759).
When designing and optimizing the performance of C-ARQ protocols, a fundamental issue is to efficiently coordinate the access to the wireless medium of the many potential network nodes (i.e., relays) that can be involved in the cooperative phase. In fact, the set of active cooperative nodes compete and contend to retransmit the packet towards the destination. In this context, an adequate Medium Access Control (MAC) protocol is crucial to enable an effective exploitation of cooperation by reducing latency and collisions. In [3], we have recently proposed Persistent Relay Carrier Sensing Multiple Access (PRCSMA), which is a new CSMA-based MAC protocol specifically designed to coordinate the retransmissions from the relays in a C-ARQ scheme. In [3] and [4], we have shown that PRCSMA can significantly outperform non-cooperative ARQ protocols. However, the assessment of the performance of this protocol has been made by considering simplified physical layer and channel models, which, on the other hand, play a crucial role to understand the actual performance of any protocol. In fact, many recent papers have clearly shown that oversimplifying the wireless channel for protocol design might eventually provide solutions that simply do not work in practical operating conditions (see, e.g., [5]- [9]).

More specifically, recent simulations [8], [9] and experimental [6] studies have clearly pointed out that shadow-fading is the crucial impairment to be considered for the analysis and design of protocols for cooperative wireless networks. In particular, in this context, including shadowing requires to carefully consider three fundamental issues:

- Non-ergodicity - Unlike fast-fading, shadowing is, in general, a non-ergodic process for the duration of a communication that is composed by the transmission of many packets [10], [11]. In other words, shadowing might change during the duration of a communication, but the fluctuations are not fast enough to go through all the states of the distribution. This implies that the most suitable metric for design, optimization, and analysis of communication protocols in the presence of shadowing is the Outage Packet Error Probability (OPEP), as opposed to the Average Packet Error Probability (APEP) [10].

- Mobility - Unlike conventional cellular systems, in cooperative networks all the nodes might be in motion. This yields fairly different propagation channel models. Recent results have shown that shadowing time-correlation plays an important role to assess the performance of multi-hop mobile networks [12], as several consecutive packets might experience the same attenuation and, therefore, might be in outage for a long period of time. In this context, it is crucial to characterize second-order statistics for a sound system design [13].

- Spatial topology - Unlike non-cooperative protocols, 


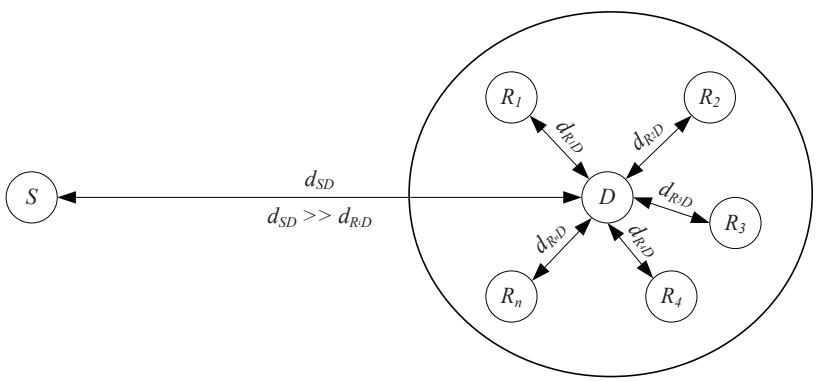

Fig. 1. System model.

when spatially-close neighbor nodes are willing to collaborate it is important to consider that radio links that are geographically close to one another often experience similar environmental shadowing effects, and, thus, might have correlated shadow-fading [6]. In these scenarios, neglecting the correlation among the cooperative links might overestimate the end-to-end performance [5].

This paper is a first attempt to include all the above issues into the analysis, design, and optimization of cooperative MAC protocols. Due to space constraints, we focus our attention on only the first problem, i.e., replacing averages with outages, and postpone the analysis of time- and spatial-correlation of shadow-fading to future research. More specifically, our contribution is the following: i) by exploiting the frameworks proposed in [11, Sec. 2.4], we provide a closed-form analytical framework to choose the subset of active relays of a C-ARQ protocol for a given Quality-of-Service (QoS) requirement imposed at the application layer (i.e., the APEP in this paper); and ii) by using this physical layer driven relay selection mechanism, we provide an analytical framework to compute the throughput of the PRCSMA protocol, as well as study the potential benefits of cooperation and the role played by random fluctuations of the received signal caused by shadowing.

The reminder of this paper is organized as follows. In Section II, system model, channel model, and PRCSMA protocol are introduced. In Section III, the problems related to introducing shadowing in our system model are described, and an adequate relay selection mechanism is proposed. In Section IV, the performance of the PRCSMA protocol is studied in closed-form. In Section V, some numerical results are shown to validate our analytical derivation and claims. Finally, Section VI, concludes the paper.

\section{SySTEM MODEL}

In this paper, we consider the system model in Fig. 1, which can be encountered in many real applications, such as a base station communicating with subscribers, a sink node connected to a sensor network, or an access point in a wireless local area network. The scenario is composed by a source $(S)$ node which wishes to establish a communication with a destination $(D)$ node. The message that $S$ wants to send to $D$ is composed by many packets, which are transmitted in different time slots. The communication ends when all the packets are delivered to $D$. Also, we assume a cooperative networking scenario in which $n$ relay nodes $\left(R_{i}\right.$ for $\left.i=1,2, \ldots, n\right)$ are willing to help $S$ to deliver, with high reliability, the packets to $D$. In particular, we consider a cooperative networking setup where the source-to-destination distance $\left(d_{S D}\right)$ is much larger than the relay-to-destination distance $\left(d_{R_{i} D}\right)$. In this scenario, if all the nodes use the same transmission power, the relay-todestination links will be, on average, of better quality than the source-to-destination link because of the shorter distance.

\section{A. Channel Model}

Let $l$ be the generic $l$-th wireless link of the network in Fig. 1. In particular, $l$ can be the source-to-destination link $(l=S D)$ or a relay-to-destination link $\left(l=R_{i} D\right.$ for $\left.i=1,2, \ldots, n\right)$. The receive-power on this link is [11, Eq. (1.1)] $P_{r}^{(l)}=$ $\left(P_{t} / d_{l}^{\alpha}\right)\left(h_{\text {fast }}^{(l)}\right)^{2} h_{\text {shad }}^{(l)}$, where: i) $P_{t}$ is the transmit-power that is common to all the nodes; ii) $\alpha$ is the path-loss coefficient; iii) $h_{\text {fast }}^{(l)}$ is the envelope of the fast-fading; iv) and $h_{\text {shad }}^{(l)}$ is the shadow-fading. Thus, the channel model is quite general and accounts for all relevant terms that are usually estimated from measurements [5]. In particular, we assume that $h_{\text {fast }}^{(l)}$ is a Nakagami- $m$ random variable with fading parameters $m_{l} \geq 0.5$ and, without loss of generality ${ }^{1}, \mathrm{E}\left\{\left(h_{\text {fast }}^{(l)}\right)^{2}\right\}=1$ for all the wireless links [11]. Also, $\tilde{h}_{\text {shad }}^{(l)}=\left(P_{t} / d_{l}^{\alpha}\right) h_{\text {shad }}^{(l)}$ denotes the random variable including path-loss and shadowing. As substantiated by many experimental measurements [11], it is well-modeled by a log-normal random variable with parameters, in $\mathrm{dB}$, equal to $\mu_{l}=\mathrm{E}\left\{10 \log _{10}\left(\tilde{h}_{\text {shad }}^{(l)}\right)\right\}$ and $\sigma_{l}^{2}=\mathrm{E}\left\{\left(10 \log _{10}\left(\tilde{h}_{\text {shad }}^{(l)}\right)-\mu_{l}\right)^{2}\right\}$. Given the system model in Fig. 1, it is reasonable to assume: i) $m_{l}=m$ and $\sigma_{l}^{2}=\sigma^{2}$ for each wireless link; ii) $\mu_{S D}=\mu_{S R_{i}}$ for $i=1,2, \ldots, n$; and iii) $\mu_{R_{i} D}=\mu_{R D}$ for $i=1,2, \ldots, n$. We emphasize that, in general, $\mu_{S D} \neq \mu_{R D}$ because source-to-destination and relay-to-destination distances are different. Finally, since, as mentioned in Section I, in this paper we are interested neither in time- nor in spatial-correlation of shadowing but only on the importance of considering it as a non-ergodic process, it is reasonable to assume that fading and shadowing are both timeand spatial-uncorrelated during the overall communication.

\section{B. PRCSMA Protocol}

In this paper, we are interested in studying the performance of the PRCSMA protocol in [3], which has been specifically designed to coordinate the channel access of the relay nodes in C-ARQ schemes. The interested reader can find the details of this protocol in [3]. In this section, we summarize only the most important aspects useful to highlight the impact of shadowing on its performance. Furthermore, we emphasize that, with very few changes, the considerations in this section are applicable to any C-ARQ based MAC protocol.

PRCSMA is based on the Distributed Coordination Function (DCF) of the IEEE 802.11 standard for wireless local area networks [3]. In particular, it is a CSMA-based protocol, where the relays sense the channel for a Distributed Inter Frame Space (DIFS) before attempting to seize it. A slotted-time reference is considered, and the collision resolution algorithm relies on a binary exponential backoff with a Contention Window $(\mathrm{CW})$ of size $\mathrm{CW}_{i}=\mathrm{CW}_{\min } \cdot 2^{i}$ for $i=1,2, \ldots, m_{b}$, where $m_{b}$ is the maximum backoff period.

\footnotetext{
${ }^{1} \mathrm{E}\{\cdot\}$ is the expectation operator.
} 
PRCSMA works as follows. For each packet sent by the source to the destination, two events can take place: 1) if the received packet is of sufficient quality to meet the QoS requirement set by the application ${ }^{2}$, then it is kept and considered as a correct packet that can be further processed by the upper layers. In that case, an ACK (acknowledgment) packet is broadcast so that relays and source can flush their cooperative and data buffers, respectively, and the communication ends. According to the rules of the DCF, the transmission of the ACK is performed after a Short Inter Frame Space (SIFS), which is needed to process the data and to compensate different propagation delays; 2) on the other hand, if the packet is of not sufficient quality, then the destination broadcasts a Call for Cooperation (CFC) packet to the nearby relay nodes to invite them to the cooperation phase. In that case, all the relay nodes that have overheard (with sufficient quality to meet the QoS requirement set by the application) the packet transmitted from the source become active relays and attempt to forward the packet to the destination. When this happens, the active relays will contend the access to the channel for relaying. More specifically, when an active relay seizes the channel to retransmit, if its packet is received by the destination with sufficient quality, then the same operations as in 1) are executed. On the other hand, if the packet is received by the destination with insufficient quality, then the destination waits for another active relay. The procedure is iterated until either a packet of sufficient quality is received and an ACK can be broadcast or a time out period has elapsed. In this latter case, the packet is discarded for the benefit of backlogged data.

\section{ImPACT OF Shadowing ON PROTOCOL DESIGN}

In this section, we aim at providing some guidelines to optimize the performance of the PRCSMA protocol to explicitly meet the QoS requirement of the application. More specifically, we are interested in providing a formal definition for "packet of sufficient quality", which determines the set of active relays along with the correct reception of a packet by the destination. As foreseen by many communication systems, we consider a QoS requirement defined in terms of packet error probability, and denote the target value by $P_{p}^{*}$. Therefore, a packet will be accepted by the MAC layer as long as the APEP is below $P_{p}^{*}$.

\section{A. Averages vs. Outages}

To better understand the role played by log-normal shadowing, let us give a closer look at the operation of the system without and with shadowing. For a more comprehensive description, the interested reader might consult [10] and [11].

- Without Shadowing. From the channel model in Section II-A, the receive-power on the $l$-th link reduces to $P_{r}^{(l)}=$ $\left(P_{t} / d_{l}^{\alpha}\right)\left(h_{\text {fast }}^{(l)}\right)^{2}$. In this case, it is reasonable and common practice to assume that fast-fading is fast enough to be modeled as an ergodic process for the duration of a packet, such that the performance can be characterized through the APEP (where the average is computed over the distribution of the fast-fading). Thus, for a given physical layer setup, the

\footnotetext{
${ }^{2}$ In Section III, we provide a formal definition of "packet of sufficient quality" by taking into account the channel model introduced in Section II-A
}

APEP is a monotonic increasing function of only the distance $d_{l}$, and we can write $\mathrm{APEP}_{l}=f\left(d_{l}\right)$ [11]. Accordingly, for a given distance the APEP is fixed, and a QoS requirement on the APEP, i.e., $\mathrm{APEP}_{l} \leq P_{p}^{*}$, turns out to be equivalent to a maximum communication range, i.e., $\operatorname{APEP}_{l} \leq P_{p}^{*} \Rightarrow d_{l} \leq$ $d_{l}^{*}$. This reduces to the well-known circular coverage model, which has extensively been shown to be inadequate for the analysis and design of communication protocols [5].

- With Shadowing. In the presence of shadowing, things change substantially. From Section II-A, the receive-power on the $l$-th link depends on $h_{\text {shad }}^{(l)}$ as well, which is a slowlyvarying random variable that can be reasonably assumed to be almost constant for the duration of a packet, such that it can experience only a few channel states for the overall duration of the communication [14, see figure in slide 6]. In other words, unlike $h_{\text {fast }}^{(l)}$, the process $h_{\text {shad }}^{(l)}$ cannot be assumed to be ergodic for the duration of the communication. In that case, we are allowed to compute the average of the packet error probability over fast-fading, but we are not allowed to do the same over shadowing. Thus, for a given physical layer setup, the APEP is a function of both the distance $d_{l}$ and the shadowing. With the notation in Section II-A, we have $\mathrm{APEP}_{l}=f\left(d_{l}, h_{\text {shad }}^{(l)}\right)=$ $f\left(\tilde{h}_{\text {shad }}^{(l)}\right)$. Thus, a QoS requirement on the APEP, i.e., $\mathrm{APEP}_{l} \leq P_{p}^{*}$, turns out to be equivalent to $\mathrm{APEP}_{l} \leq$ $P_{p}^{*} \Rightarrow \tilde{h}_{\text {shad }}^{(l)} \geq \gamma_{l}^{*}$ [11], where $\gamma_{l}^{*}$ is a threshold value that depends on the physical layer setup. Further details about $\gamma_{l}^{*}$ are given in Section III-B. However, even though shadowfading is non-ergodic and we cannot compute the average over its distribution, $\tilde{h}_{\text {shad }}^{(l)}$ is still a random variable with log-normal distribution. So, the quality of the link cannot be characterized only by the proximity (distance) between the nodes, but needs to be characterized statistically. In this case, the most suitable metric is the OPEP [11], i.e., the probability of receiving a packet of insufficient quality, which is defined as ${ }^{4}$ $\mathrm{OPEP}_{l}=\operatorname{Pr}\left\{\mathrm{APEP}_{l} \geq P_{p}^{*}\right\}=\operatorname{Pr}\left\{f\left(\tilde{h}_{\text {shad }}^{(l)}\right) \geq P_{p}^{*}\right\}=$ $\operatorname{Pr}\left\{\tilde{h}_{\text {shad }}^{(l)} \leq \gamma_{l}^{*}\right\}=Q\left(\left[\mu_{l}-10 \log _{10}\left(\gamma_{l}^{*}\right)\right] / \sigma\right)$, where the last identity follows from the assumption that $\tilde{h}_{\text {shad }}^{(l)}$ is lognormal distributed.

From the considerations above, we are now ready to define in a formal way the concept "packet of sufficient quality" when shadowing is taken into account. In particular, a packet is accepted by the MAC layer if and only if the mean receivepower is above the threshold $\gamma_{l}^{*}$. In fact, both relays and destination can measure the mean receive-power, compare it with the pre-assigned threshold $\gamma_{l}^{*}$, and decide whether the relay can become active and whether the destination should request a retransmission. With this policy, and from the definition of $\gamma_{l}^{*}$ above, i.e., $\gamma_{l}^{*}=f^{-1}\left(P_{p}^{*}\right)$, we can guarantee that if the mean receive-power is above $\gamma_{l}^{*}$, then the packet is decoded with $\mathrm{APEP}_{l} \leq P_{p}^{*}$, which allows us to meet the QoS requirement. Furthermore, since $\tilde{h}_{\text {shad }}^{(l)}$ is a random variable on its own, we can characterize statistically the quality of a link by exploiting the OPEP, which yields the

\footnotetext{
${ }^{3} f(\cdot)$ denotes "function of".

${ }^{4} Q(x)=(1 / \sqrt{2 \pi}) \int_{x}^{+\infty} \exp \left(-t^{2} / 2\right) d t$ is the Q-function, and $\operatorname{Pr}\{\cdot\}$ denotes probability.
} 
probability that a packet is of not sufficient quality by taking into account the random nature of the shadowing. It is usual to say that a link is in outage when $\tilde{h}_{\text {shad }}^{(l)} \leq \gamma_{l}^{*}$. Thus, the set of active relays introduced in Section II-A is given by only those relays which are not in outage, and the simple formula $P_{l}^{\text {active }}=1-\mathrm{OPEP}_{l}=1-Q\left(\left[\mu_{l}-10 \log _{10}\left(\gamma_{l}^{*}\right)\right] / \sigma\right)$ provides a closed-form expression of the probability that a link is active by taking into account fast-fading, shadowing, and a realistic physical layer setup.

\section{B. Computation of $\gamma_{l}^{*}$ for a Realistic Physical Layer}

The crucial point in the relay selection procedure described above in the presence of shadowing is the closed-form computation of $\gamma_{l}^{*}=f^{-1}\left(P_{p}^{*}\right)$, by explicitly taking into account the most important parameters of the physical layer. The computation of this threshold for a generic physical layer is not a trivial task, especially when the QoS requirement of interest is the APEP, i.e., $\mathrm{APEP}_{l} \leq P_{p}^{*}$. This is an important contribution of this paper, as this threshold is often set to arbitrary values that do not account for the actual physical layer implemented by the communication system, such as modulation, coding, packet length, etc. To this end, we exploit the methodology recently proposed in [11].

As a realistic illustrative example, at the physical layer we assume: i) single-input-single-output transceivers for all the nodes of the network in order to comply the with lowcomplexity requirement of the nodes, which exploit distributed rather than co-located multiple-antenna diversity; ii) a general Multilevel Phase Shift Keying (M-PSK) modulation scheme; iii) a channel block-code; iv) hard-decision decoding; and v) ideal interleaving. Furthermore, we define a packet error as the probability that at least one bit of the packet is wrong. With these assumptions, $\gamma_{l}^{*}=f^{-1}\left(P_{p}^{*}\right)$ can be computed in closed-form as follows [11, Sec. 2.4]:

$$
\gamma_{l}^{*}=\frac{m}{g_{\bmod }}\left\{\left[\frac{P_{b}^{*}}{k_{\bmod }} \cdot \sqrt{1-\tilde{t} \cdot\left(\sqrt[m]{\frac{P_{b}^{*}}{k_{\bmod }}}\right)}\right]^{-(1 / m)}-1\right\}
$$

where we have defined:

$P_{b}^{*}=\left\{\frac{P_{c}^{*} \cdot(t+1) \cdot B(t+1, J-t)}{\left\{1-\tilde{y}\left[P_{c}^{*} \cdot(t+1) \cdot B(t+1, J-t)\right]^{(t+1)^{-1}}\right\}^{J-t-1}}\right\}^{(t+1)^{-1}}$ and: i) $P_{c}^{*}=1-\left(1-P_{p}^{*}\right)^{k / N}$; ii) $g \bmod =$ $\sin ^{2}(\pi / M)$; iii) $\tilde{t}=m /(m+1)$; iv) $k_{\bmod }=$ $\Gamma(m+0.5) /\left[\sqrt{\pi} \max \left\{\log _{2}(M), 2\right\} \Gamma(m+1)\right] ; \quad$ v) $\tilde{y}=$ $(t+1) /(t+2)$; vi) $B(x, y)=[\Gamma(x) \Gamma(y)] / \Gamma(x+y)$; vii $)$ $\Gamma(z)=\int_{0}^{+\infty} \xi^{z-1} \exp (-\xi) d \xi$ is the Gamma function; viii) $M$ is the modulation order of M-PSK; ix) $N$ is the number of bits in a packet; $\mathrm{x}) k$ is the number of information bits in a codeword; $x i$ ) $J$ is the number of bits (information and redundancy) in a codeword; and xii) $t$ is the number of errors the channel code can correct.

It is worthwhile mentioning that, in spite of the generality of the physical layer we consider, the final expression in (1) is very simple to compute, and it only depends on the fading parameters rather than on instantaneous channel state information. This means that it can be computed during the initial setup and used for the whole duration of the communication.
Also, we emphasize that in our system model we have $m_{l}=m$ for all the wireless links, which implies that $\gamma^{*}=\gamma_{l}^{*}$ is the same for all the wireless links. Finally, we note that the QoS requirement, $P_{p}^{*}$, is explicitly given in (1).

\section{Closed-Form Performance of PRCSMA}

Having defined that most appropriate performance metric to take into account the effect of shadowing, and provided a relay selection mechanism that accounts for the QoS requirement requested by the application for a realistic physical layer and channel model, we are now ready to analyze the performance of the PRCSMA protocol. In particular, we focus our attention on the analysis of the throughput with the aim of understanding the improvement introduced by cooperative networking and the number, $n$, of possible active relays.

The average throughput of the system, expressed in bits per second (bps), can be computed as the ratio between the average size of the data packets, i.e., $\mathrm{E}[L]$, and the average total time required to transmit a packet (by including transmission time, MAC overhead, and possible retransmissions). Accordingly, the throughput, $S$, of the system can be expressed as:

$$
S[\mathrm{bps}]=\frac{\mathrm{E}\{L\}\left(1-\mathrm{OPEP}_{S D} \cdot p_{n R}\right)}{\left(1-\mathrm{OPEP}_{S D}\right) T_{S}+\mathrm{OPEP}_{S D} \cdot \mathrm{E}\left\{T_{S \mid \mathrm{COOP}}\right\}}
$$

where: i) in our case $\mathrm{E}\{L\}=N$, as we consider the transmission of packets with a constant number of bits; ii) $\mathrm{OPEP}_{S D}$ is the outage packet error probability that can be obtained from Section III-A; iii) $p_{n R}$ is the probability that there are no active relays during the cooperation phase or, in other words, that all the relays are in outage. Accordingly, we have $p_{n R}=\mathrm{OPEP}_{S D}^{n}$; iv) $T_{S}$ is the duration of the transmission of a single packet; and v) $\mathrm{E}\left\{T_{S \mid \mathrm{COOP}}\right\}$ is the average duration of a packet transmission when cooperation is requested by the destination. Furthermore, it is worthwhile mentioning that the substraction in the numerator takes into account the fraction of packets that are discarded after cooperation when all the relays are in outage, and, thus, there are no active relays that can help. Also, the denominator is a weighted sum of the probability that the source-to-destination link is in outage.

Finally, an important consideration is worth being made about (3) to emphasize, once again, the difference of the analysis in the presence of shadowing. By carefully looking at previous analysis neglecting shadowing [3], [4], we notice that in (3) the APEP over the source-to-destination link is actually replaced by the OPEP over the same link. This confirms that in the presence of shadowing the metric that should be used to characterize the performance of the protocol is the outage probability rather than the average error probability. More specifically, it would have been erroneous to replace in [3], [4] the PEP averaged over both fast-fading and shadowing because, as explained in Section III-A, shadowing is a nonergodic process for the duration of the communication, and, thus, it does not experience all the fading states (see, e.g., [14, figure in slide 6] and [10] for further comments).

To compute (3), we need a closed-form expression of $\mathrm{E}\left\{T_{S \mid \mathrm{COOP}}\right\}$. This parameter can be obtained by using considerations similar to [3], [4], but updating the analytical development to incorporate the notion of outage instead of average. Due to space constraints, we are unable to report all 
the details of the analytical derivation and only summarize the final result. In particular, $\mathrm{E}\left\{T_{S \mid \mathrm{COOP}}\right\}$ can be computed as:

$$
\left\{\begin{array}{l}
\mathrm{E}\left\{T_{S \mid \mathrm{COOP}}\right\}=T_{\mathrm{CFC}}+T_{\mathrm{ACK}}+2 T_{\mathrm{SIFS}}+T_{\mathrm{COOP}} \\
T_{\mathrm{COOP}}=p_{n R} T_{\mathrm{TO}-\mathrm{COOP}}+\left(1-p_{n R}\right) \mathrm{E}\left\{T_{\mathrm{CONT}}\right\}
\end{array}\right.
$$

where: i) $T_{\mathrm{CFC}}$ is the transmission time of the CFC packet sent by the destination to initiate the cooperation phase; ii) $T_{\mathrm{ACK}}$ is the transmission time of the ACK related to the CFC packet; iii) $T_{\text {SIFS }}$ is the duration of the SIFS that is used to compensate for the propagation delays; iv) $T_{\mathrm{TO}-\mathrm{COOP}}$ is the time-out after which the cooperation phase ends if no retransmissions from the relays are received; and v) $\mathrm{E}\left\{T_{\mathrm{CONT}}\right\}$ is the average contention time due to the PRCSMA protocol. It can be computed by resorting to the procedure described in [4] and by considering the actual behavior of the new protocol based on outages. More specifically, only two parameters need to be replaced in the framework in [4], i.e., the average number of active relays, i.e., $\mathrm{E}\{R\}$, and the average number of transmissions from all relays to deliver a packet to the destination, i.e., $\mathrm{E}\{K\}$. By taking into account the considerations in Section III-A, they can be computed in closed-form as follows:

$$
\begin{aligned}
\mathrm{E}\{R\}=\sum_{r=0}^{n}\{r & {\left.\left[\left(\begin{array}{l}
n \\
r
\end{array}\right)\left(1-\mathrm{OPEP}_{S D}^{r}\right) \operatorname{OPEP}_{S D}^{n-r}\right]\right\}=n \mathrm{OPEP}_{S D} } \\
\mathrm{E}\{K\} & =\sum_{k=0}^{+\infty}\left[(k+1) \mathrm{OPEP}_{R D}^{k}\left(1-\mathrm{OPEP}_{R D}\right)\right] \\
& =\left(1-\mathrm{OPEP}_{R D}\right)^{-1}
\end{aligned}
$$

where $(\cdot)$ is the binomial coefficient, and the last identities are obtained with simple algebraic manipulations.

\section{Numerical Results}

The aim of this section is twofold: i) to validate the accuracy of the analytical frameworks developed in Section III for the physical layer and in Section IV for the MAC protocol; and ii) to analyze the impact of shadowing and cooperation on the performance of the overall PRCSMA protocol. The analytical results are compared to Monte Carlo simulations by applying all the rules of the protocol without any simplifications. The following set of parameters is used to obtain the presented figures. The physical layer parameters are: i) $M=8$; ii) $m=3$; iii) Hamming block-code with $J=7, k=4$, and $t=1$; iv) $N=128$ bytes; and v) $\mu_{R D}=40 \mathrm{~dB}$. Furthermore, the QoS requirement is set to $P_{p}^{*}=0.1$, which yields a threshold equal to $\gamma^{*}=41.12=16.14 \mathrm{~dB}$. Note that these parameters are common to all links, and thus we are considering that the relays use all the same transmission rate. The MAC layer parameters are: i) SlotTime $=10 \mu \mathrm{s}$; ii) the minimum and maximum values of $\mathrm{CW}$ are $\mathrm{CW}_{\min }=16$ and $\mathrm{CW}_{\mathrm{MAX}}=32$, respectively; iii) the overall duration of MAC header and physical-layer preamble is $232 \mu \mathrm{s}$; iv) DIFS $=50 \mu \mathrm{s} ; \mathrm{v})$ SIFS $=10 \mu \mathrm{s}$; vi) the length of RTS (Request-to-Send) packets is 20 bytes; and vii) the lengths of CTS (Clear-to-Send), CFC, and ACK packets are 14 bytes each.

In Fig. 2 and Fig. 3, we show the normalized throughput, i.e., $S_{\text {norm }}=S /\left(T_{s} N\right)$ for $n=1$ and $n=10$, respectively. We notice a very good agreement between Monte Carlo simulations and the analytical models developed in Section III and in Section IV. As far as the system behavior is

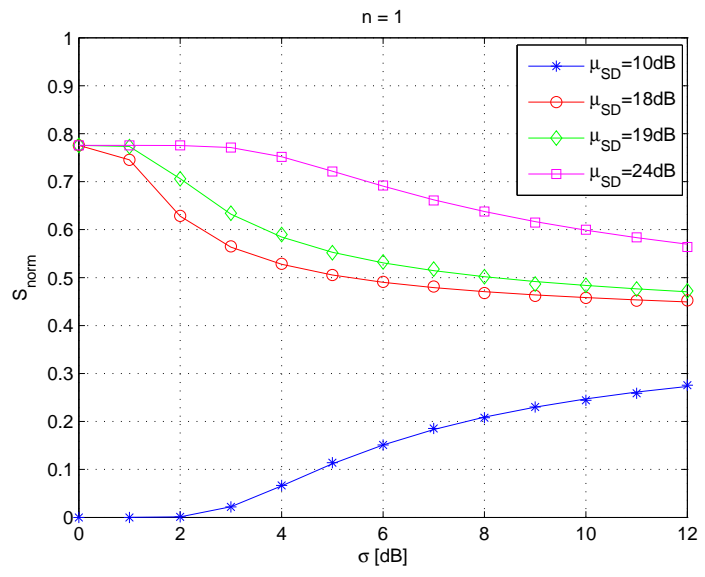

Fig. 2. Normalized throughput against the quality of the source-to-destination link $\left(\mu_{S D}\right)$ and the severity of shadowing $(\sigma)$. Solid lines show the analytical framework and markers show Monte Carlo simulations $(n=1)$.



Fig. 3. Normalized throughput against the quality of the source-to-destination link $\left(\mu_{S D}\right)$ and the severity of shadowing $(\sigma)$. Solid lines show the analytical framework and markers show Monte Carlo simulations $(n=10)$.

concerned, the following conclusions can be drawn: i) $S_{\text {norm }}$ increases when the quality of the source-to-destination link improves (i.e., $\mu_{S D}$ increases), as expected; ii) if $\mu_{S D} \geq$ $18 \mathrm{~dB}$, then $S_{\text {norm }}$ decreases when the severity of the shadowfading increases (i.e., $\sigma$ increases). On the other hand, $S_{\text {norm }}$ increases if $\mu_{S D}=10 \mathrm{~dB}$. This behavior can be explained by taking into account the working principle of the cooperative network under analysis and the system setup. In fact, if $\mu_{S D}<\gamma^{*}=16.14 \mathrm{~dB}$ the transmit-power is too low to even offset the deterministic path-loss caused by the transmission distance. In this case, the system is almost always in outage and the throughput is very low. However, if $\sigma$ increases, there are occasional situations in which shadowing results in a constructive gain, which yields a higher throughput than the scenario with no shadowing (i.e., $\sigma=0 \mathrm{~dB}$ ). In this case, shadowing is beneficial. However, we emphasize that a system in good operation should, at least, be designed to have a receive-power that guarantees a good communication in the absence of shadowing, i.e., $\mu_{S D} \geq \gamma^{*}$ in our case. These two trends highlight the important role played by shadowing to assess the performance of MAC protocols, as well as that severe shadowing conditions $(\sigma=12 \mathrm{~dB})$ might reduce the throughput by up to $37 \%$ when $\mu_{S D}>\gamma^{*}$; and iii) the throughput increases with the number of potential relays $n$.

In Fig. 4 and Fig. 5, we study the gain introduced by 


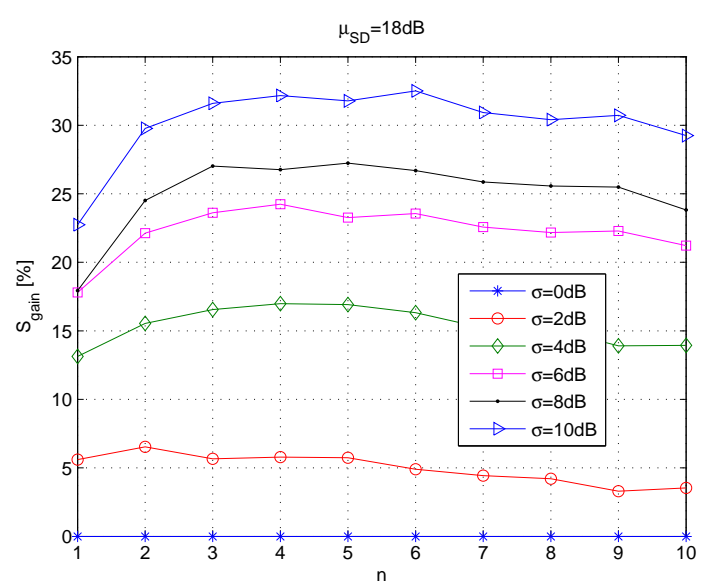

Fig. 4. Throughput gain against the number of potential relays $(n)$ and the severity of shadowing $(\sigma)$ when $\mu_{S D}=18 \mathrm{~dB}$.

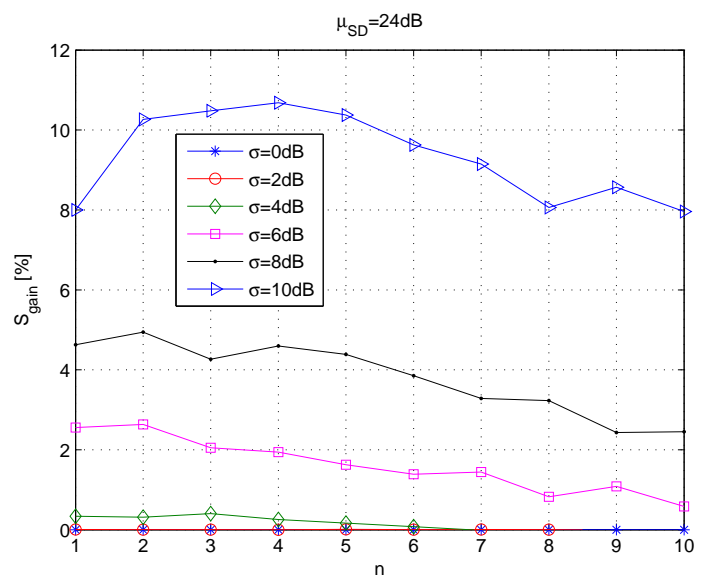

Fig. 5. Throughput gain against the number of potential relays $(n)$ and the severity of shadowing $(\sigma)$ when $\mu_{S D}=24 \mathrm{~dB}$.

cooperation for different shadowing conditions, when $\mu_{S D}=$ $18 \mathrm{~dB}$ and $\mu_{S D}=24 \mathrm{~dB}$, respectively. The main aim is to understand if there is an optimal value of $n$ to achieve the best throughput gain by exploiting the cooperative networking paradigm. To this end, we plot the normalized throughput gain with respect to the setup with no cooperative nodes, i.e., $n=0$. In formulas, we have $S_{\text {gain }}[\%]=$ $100 \times\left\{\left[S_{\text {norm }}(n)-S_{\text {norm }}(n=0)\right] / S_{\text {norm }}(n=0)\right\}$. The results are very interesting, and three main conclusions can be drawn: i) there is an optimal value of $n$ to achieve the best throughput. In particular, too many potential relays result in a higher overhead, which reduces the net throughput; ii) the gain of cooperation is higher for more severe shadowing conditions (i.e., higher values of $\sigma$ ). In fact, cooperation has primarily been introduced to counteract severe shadowing through distributed diversity. Thus, the gain of cooperation in the absence of shadowing is close to zero, since we can always deterministically set the transmit-power for a fixed distance and avoid outages. This highlights, once again, the important role of shadowing for the analyzed cooperative scenario; and iii) the throughput gain introduced by cooperation increases for worse source-to-destination links (i.e., $\mu_{S D}$ decreases). This is an expected result, and shows that cooperation should be used only when actually needed, i.e., when the direct link is of not sufficient quality. The PRCSMA protocol is actually designed to account for that, as the cooperation phase is initiated only if this link fails to deliver the packets, thus controlling the overhead when cooperation is not needed.

\section{CONCLUSION}

In this paper, we have proposed an analytical methodology for the analysis and design of cooperative MAC protocols with a realistic physical layer and wireless channel model. Notably, we have carefully addressed the issues related to accounting for slowly-varying shadow-fading in the analysis and design of C-ARQ based MAC protocols. We have proved that the appropriate performance metric to be considered is the outage error probability, as opposed to usual practice of considering either just the propagation distance or the error probability averaged over fast-fading. Also, we have studied the PRCSMA protocol, and have highlighted that shadowing can significantly affect its performance, as well as that cooperation can substantially improve the end-to-end performance. Ongoing research is concerned with the extension of the proposed framework to take into account time- and, especially, spatial-correlation of shadow-fading in the analysis and design of C-ARQ based MAC protocols.

\section{REFERENCES}

[1] M. Zorzi, R. R. Rao, and L. B. Milstein, "ARQ error control for fading mobile radio channels," IEEE Trans. Vehicular Technol., vol. 46, no. 2, pp. 445-455, May 1997.

[2] M. Dianati, X. Ling, K. Naik, and X. Shen, "A node-cooperative ARQ scheme for wireless ad hoc networks," IEEE Trans. Vehicular Technol., vol. 55, no. 3, pp. 1032-1044, May 2006.

[3] J. Alonso-Zárate, E. Kartsakli, Ch. Verikoukis, and L. Alonso, "Persistent RCSMA: A MAC protocol for a distributed cooperative ARQ scheme in wireless networks," EURASIP J. Adv. Sig. Process., vol. 2008, May 2008.

[4] J. Alonso-Zárate, L. Alonso, and Ch. Verikoukis, "Performance analysis of a persistent relay carrier sensing multiple access protocol," IEEE Trans. Wireless Commun., vol. 8, no. 12, pp. 5827-5831, Dec. 2009.

[5] C. Newport, D. Kotz, Y. Yuan, R. S. Gray, J. Liu, and C. Elliott, "Experimental evaluation of wireless simulation assumptions," Simulation, vol. 83, no. 9, pp. 643-661, Sept. 2007. [Online]. Available: http://cmc.cs.dartmouth.edu/cmc/papers/kotz:axioms-tr2.pdf.

[6] P. Agrawal and N. Patwari, "Correlated link shadow fading in multihop wireless networks," IEEE Trans. Wireless Commun., vol. 8, no. 8, pp. 4024-4036, Aug. 2009.

[7] B. H. Elyes, G. Chelius, and J.-M. Gorce, "Impact of the physical layer modeling on the accuracy and scalability of wireless network simulation," Simulation, vol. 85, no. 9, pp. 574-588, Sept. 2009.

[8] L. Hanzo II and R. Tafazolli, "The effects of shadow-fading on QoS-aware routing and admission control protocols designed for multi-hop MANETs," Wireless Commun. Mob. Comput., vol. 11, no. 1, pp. 1-22, Jan. 2011.

[9] L. Hanzo II and R. Tafazolli, "QoS-aware routing and admission control in shadow-fading environments for multi-rate MANETs," IEEE Trans. Mob. Comput., vol. 10, no. 5, pp. 622-637, May 2011.

[10] P. Mary, M. Dohler, J.-M. Gorce, G. Villemaud, and M. Arndt,, "Mary symbol error outage over Nakagami- $m$ fading channels in shadowing environments," IEEE Trans. Commun., vol. 57, pp. 2876-2879, Oct. 2009.

[11] P. Mary, "Etude analytique des performances des systèmes radio-mobiles en présence d'évanouissements et d'effet de masque," Ph.D. dissertation, Institut des Sciences Appliquées de Lyon, Feb. 2008. [Online]. Available: http://docinsa.insa-lyon.fr/these/2008/mary/these.pdf.

[12] Z. Wang, E. K. Tameh, and A. R. Nix, "Joint shadowing process in urban peer-to-peer radio channels," IEEE Trans. Vehicular Technol., vol. 57, no. 1, pp. 52-64, Jan. 2008.

[13] M. Di Renzo, L. Imbriglio, F. Graziosi, and F. Santucci, "Secondorder statistics of amplify-and-forward multi-hop wireless networks: A framework for computing the end-to-end SNR auto-correlation function over log-normal shadowing channels," IEEE ICUMT, pp. 1-6, Oct. 2009.

[14] P. Mary, "Reliability of radio-mobile systems considering fading and shadowing channels," presentation given at EURECOM, France, Jan. 2009. [Online]. Available: http://www.eurecom.fr/util/seminairedownload.fr.htm?id=288. 